\title{
Knockdown of IncRNA UCAI inhibits proliferation and invasion of papillary thyroid carcinoma through regulating miR-204/IGFBP5 axis
}

This article was published in the following Dove Press journal:

OncoTargets and Therapy

\author{
Hongyu Liu',* \\ Ruil $\mathrm{Li}^{2, *}$ \\ Lianyue Guan' \\ Tao Jiang' \\ 'Department of \\ Hepatopancreatobiliary Surgery, \\ China-Japan Union Hospital of \\ Jilin University, Nangun District, \\ Changchun 130033, China; \\ ${ }^{2}$ Department of Thyroid Surgery, \\ The First Hospital of Jilin University, \\ Chaoyang District, Changchun \\ |3002 I, China \\ *These authors contributed equally \\ to this work
}

Background: Long noncoding RNA (LncRNA) UCA1 has been reported to function as an oncogene in multiple cancers. However, the biological roles and underlying mechanism of UCA1 in papillary thyroid carcinoma (PTC) remain unclear. This study aimed to investigate the underlying function of UCA1 on thyroid cancer progression.

Materials and methods: A series of experiments involving Cell Counting Kit-8, woundhealing, and transwell invasion assays were conducted to determine the cellular capabilities of proliferation, migration, and invasion, respectively. Binding sites between UCA1 and miR-204 were identified using a luciferase reporter system, whereas mRNA and protein expression of target genes were determined by real-time quantitative reverse transcription-PCR (qRT-PCR) and Western blot, respectively.

Results: The results revealed that UCA1 was upregulated in PTC tissue and cell lines. UCA1 knockdown significantly suppressed the cell proliferation, migration, and invasion of TPC-1 cells. Bioinformatics analysis and luciferase reporter assay verified the complementary binding within UCA1 and miR-204 at the 3'-UTR. Moreover, miR-204 inhibition reversed the UCA1 knockdown-mediated inhibitory effect on cell proliferation, migration, and invasion. We also found that UCA1 could regulate expression of IGFBP5, a direct target of miR-204 in PTC.

Conclusion: Our study demonstrated that UCA1 exerts activity of oncogenes in PTC through regulating miR-204/IGFBP5 axis.

Keywords: papillary thyroid carcinoma, lncRNA, UCA1, miR-204, IGFBP5

\section{Introduction}

Papillary thyroid carcinoma (PTC), accounting for $\sim 90 \%$ of all thyroid cancer, is the most common endocrine malignancy in the worlds and the top 10 common cancer in Chinese female. ${ }^{1}$ After the effective and reasonable treatment, most patients with PTC have a good prognosis, and the 5-year survival rate of PTC is $\sim 90 \%{ }^{2}$ However, a small fraction of patients develop PTC with dedifferentiation, resulting in the decline in the survival rate and life quality of patients. ${ }^{3}$ Genetic markers have a high specificity for malignancy identification and can be used in operation procedure and prevention for PTC. Therefore, understanding molecular events underlying the initiation and progression of PTC is critical to develop molecular targeted for treatment of this disease.

With a growing number of long noncoding RNAs (lncRNAs) being discovered in recent years, investigation of the biological functions of lncRNAs has become an attractive research hot spot. ${ }^{4}$ IncRNAs are a large family of evolutionarily conserved noncoding RNAs longer than 200 nucleotides in length without protein-coding capacity. ${ }^{5}$ lncRNA has been reported to be involved in various cellular processes,
Correspondence: Tao Jiang

Department of Hepatopancreatobiliary Surgery, China-Japan Union Hospital of Jilin University, I26\# Xiantai Street, Nangun District, Changchun I30033, China

Email jiangtao1924@sina.com 
including chromatin dynamics, embryonic development, differentiation, and carcinogenesis. ${ }^{6,7}$ Accumulating evidence demonstrated that dysregulated lncRNAs implicated in carcinogenesis and tumor progression through regulating cell proliferation, migration, invasion, and apoptosis in a variety of tumors. ${ }^{8,9}$ A number of lncRNAs were identified to contribute to occurrence and progression of PTC, suggesting that lncRNAs might serve as a diagnosis marker and molecular therapy target. ${ }^{10,11}$

Human UCA1 is an lncRNA that was initially reported to be overexpressed in bladder cancer and serve as a biomarker for the diagnosis of bladder cancer. ${ }^{12}$ Several reports revealed that UCA1 expression was upregulated and functioned as an oncogene in breast cancer, prostate cancer, esophageal cancer, pancreatic cancer, gastric cancer, colorectal cancer, epithelial ovarian cancer, and non-small-cell lung cancer. ${ }^{13-20}$ However, the functional role of UCA1 in the PTC progression is unclear. Therefore, the aims of this study were to investigate the expression pattern, biological function, and action mechanism of UAC1 in PTC progression.

\section{Materials and methods}

\section{Tissue samples}

The human PTC tissues and the adjacent normal tissues were obtained from 42 PTC patients (mean age, 60.5 \pm 4.1 years, age range, 39.2-75.8 years; male, 14 cases, and female, 28 cases) who received surgical treatment at the First Affiliated Hospital of Jilin University. All samples were confirmed by two pathologists independently via pathological diagnosis. Patients who received the chemotherapy, radiotherapy, or other treatments were excluded. All patients whose tissues were used in this research provided written informed consent. This study was reviewed and approved by the Clinical Research Ethics Committee of Jilin University and in accordance with the ethical standards formulated in the Declaration of Helsinki and the national regulations.

\section{Cell culture}

Two PTC cell lines (BCPAP and TPC-1) and one normal thyroid cell line Nthy-ori3-1 were purchased from the Institute of Biochemistry and Cell Biology of Chinese Academy of Sciences (Shanghai, China). All cells were kept in the RPMI-1640 medium (Life Technologies, Waltham, MA, USA) containing 10\% heat-inactivated FBS (Gibco, Grand Island, NY, USA), $100 \mathrm{U} / \mathrm{mL}$ penicillin, and $100 \mathrm{mg} / \mathrm{mL}$ streptomycin in a humidified atmosphere at $37^{\circ} \mathrm{C}$ containing $5 \% \mathrm{CO}_{2}$.

\section{RNA interference and cell transfection}

Gene-specific stealth RNAi oligonucleotides (siRNA) against UCA1 (si-UCA1) and scramble negative control (si-NC) were designed and synthesized by GenePharma (Shanghai, China). The sequences of si-UCA1 and si-NC were described as follows: si-UCA1: 5'-GGACAACAGUACACGCAUATT-3' and si-NC: 5'-UUCUCCGAACGUGUCACGUTT-3'. miR-204 mimics (miR-204), negative control mimics (miR-NC), miR-204 inhibitor (anti-miR-204), and corresponding negative control (anti-miR-NC) were all purchased from GenePharma. All of these were transfected into TPC-1 cells using lipofectamine 2000 (Invitrogen, Carlsbad, CA, USA) according to the manufacturer's instructions.

\section{Total RNA extraction and quantitative reverse transcription-PCR (qRT-PCR)}

Total RNA was isolated from tissues and cultured cells using the TRIzol reagent (Invitrogen) according to the manufacturer's instructions. The concentration and purity were detected by NanoDrop ND-1000 Spectrophotometer (Thermo Scientific, Waltham, MA, USA). Then, total RNA was reversed transcribed using the First Strand cDNA Synthesis Kit (Takara, Tokyo, Japan) or TaqMan microRN (miRNA) Reverse Transcription Kit (Applied Biosystems, Waltham, MA, USA). Quantitative real-time PCR was performed with SYBR Green PCR Master Mix reagents (Takara) in the 7500 Real-Time PCR System (Applied Biosystems). The primer sequences were as follows: UCA1 forward, 5'-CCACACCCAAAACAAAAAATCT-3' and reverse, 5'-TCCCAAGCCCTCTAACAACAA-3'; IGFBP5 forward, 5'-ACGCGTCGACATGGGCTCCTTC GTGCAC-3' and reverse, 5'-CGCGGATCCATCACTCA ACGTTGCTGCTG-3'; miR-204 forward, 5'-CTGTCACTC GAGCTGCTGGAATG-3' and reverse, 5' GGAGTCGGCAATT-3'; U6 forward, 5'-CGCTTCGGCAG CACATATAC-3' and reverse, $5^{\prime}$-TTCACGAATTTGCGT GTCAT-3'; and GAPDH forward, 5'-CATGAGAAGTA TGACAACAGCCT-3' and reverse, 5'-AGTCCTTCCAC GATACCAAAGT-3'. The relative expression level of mRNA or miRNA was normalized to that of internal control GAPDH or U6. Comparative quantification was determined using the $2^{-\Delta \Delta C_{\mathrm{t}}}$ method.

\section{Cell proliferation}

The TPC- 1 cells $(50,000$ cells/well) were seeded in a 96-well plate and cultured at the DMEM with $10 \% \mathrm{FBS}$ in a humidified atmosphere at $37^{\circ} \mathrm{C}$ containing $5 \% \mathrm{CO}_{2}$. Cell proliferation abilities at 24,48 , and 72 hours after transfection 
were detected using Cell Counting Kit-8 (Dojindo, Japan) according to the manufacturer's instructions. The absorbance at $450 \mathrm{~nm}$ was then measured using a micro-plate reader (BioTek Instruments, Inc., Winooski, VT, USA).

\section{Wound-healing assay}

Cell migration was determined by wound-healing assay. Briefly, transfected cells were seeded in six-well plates $\left(5 \times 10^{4}\right.$ cells per well) and were serum starved for 24 hours at full confluence. Cells were scratched using a sterile plastic micropipette tip to create an artificial wound. Photographs were taken at 0 and 24 hours after the wounding using inverted Nikon Eclipse TS100 phase-contrast microscope (Nikon Corporation, Tokyo, Japan). Wound closure was measured using Nikon NIS-Element Basic Research v3.2 software.

\section{Transwell invasion assay}

Cell invasion potential was evaluated using transwell chambers ( $8 \mu \mathrm{m}$ pore; BD Biosciences, San Jose, CA, USA). Briefly, $2 \times 10^{5}$ transfected cells in the $200 \mu \mathrm{L}$ serum-free medium were plated into the upper chamber with Matrigel (BD Biosciences), while media containing 10\% FBS was added to the bottom chambers to attract cells. After 48 hours incubation, noninvading cells were removed from the upper chamber with a cotton swab, while invaded cells located on the lower surface of the membrane were fixed with $4 \%$ paraformaldehyde and stained with $1 \%$ crystal violet solution (Sigma-Aldrich Co., St Louis, MO, USA). The invasive cells from five selected randomly fields were counted using an inverted microscope (Leica, Malvern, PA, USA).

\section{Luciferase reporter assay}

A target prediction software starBase 2.0 was used to predict potential UCA1-miRNA interactions. The DNA fragment of the UCA1 3'-UTR containing either the predicted binding site for miR-204 or a mutated 3'-UTR was inserted into the psiCHECK2 vector (Promega, Madison, WI, USA) and named as wild-type (Wt)-UCA1 or mutant-type (Mut)UCA1, respectively. For luciferase reporter assay, TPC-1 cells were grown to $70 \%-80 \%$ confluence in $24-$ well plates $\left(5 \times 10^{4}\right.$ cells/well) and were cotransfected with the psiCHECK2 vector containing either the Wt-UCA1 or MutUCA1 3'-UTR and miR-204 mimics or miR-NC mimics using a Lipofectamine 2000 according to the manufacturer's instructions. Luciferase activity was measured at 48 hours after transfection using a dual-luciferase reporter assay system (Promega) and normalized to Renilla activity.

\section{Western blot}

Cell lysates were prepared by extracting proteins with RIPA (Beyotime, Shanghai, China). Protein concentration was quantified using the Pierce Bicinchoninic Acid Protein Assay Kit (Thermo Scientific). Equal amounts of protein (30 $\mu \mathrm{g})$ were separated by $10 \%$ SDS-PAGE and transferred to polyvinylidene difluoide (PVDF) membranes (Millipore, Bedford, MA, USA). After blocked in 5\% skim milk for 2 hours, the membranes were incubated with mouse-anti human IGFBP5 polyclonal antibody (1:500 dilution; Santa Cruz Biotechnology Inc., Santa Cruz, CA, USA) and mouseanti human GAPDH polyclonal antibody (1:2,000 dilution; Santa Cruz Biotechnology Inc.) overnight at $4^{\circ} \mathrm{C}$. Then membranes were incubated with the goat anti-mouse IgG linked to a horse radish peroxidase (Santa Cruz Biotechnology Inc.) for 2 hours at $37^{\circ} \mathrm{C}$. Protein bands were detected using enhanced chemiluminescence (ECL) reagent (Amersham, GE Healthcare, Velizy-Villacoublay, France) and exposed on an X-ray film. Endogenous control was GAPDH. Gray analysis by image analysis was performed using the software Gel-Pro Analyzer (United States Biochemical, Cleveland, $\mathrm{OH}, \mathrm{USA}$ ) after scanning. The semi-quantitative analysis was performed according to the relative expression of objective protein and internal control protein GAPDH, namely the ratio of objective protein and GAPDH.

\section{Statistical analyses}

All statistical analyses were performed with SPSS software (version 21.0; SPSS Inc., Chicago, IL, USA). The data were expressed as mean $\pm \mathrm{SD}$ of three independent experiments. Comparison between groups was performed using paired $t$-test or ANOVA. Relationships between UCA1 expression and miR-204 or IGFBP5 mRNA levels were analyzed by Pearson correlation analysis. $P<0.05$ was regarded as statistically significant.

\section{Results \\ LncRNA UCAI is upregulated in human PTC}

The expression levels of UCA1 were measured in 42 cases of human PTC tissues and adjacent noncancerous tissues by qRT-PCR. Our data revealed that UCA1 expression was upregulated in PTC tissues compared with adjacent noncancerous tissues $(P<0.01$; Figure $1 \mathrm{~A})$. To confirm this change in PTC cell lines, we measured the UCA1 expression level in two PTC cell lines (BCPAP and TPC-1) and normal thyroid cell line Nthy-ori3-1 by qRT-PCR. 

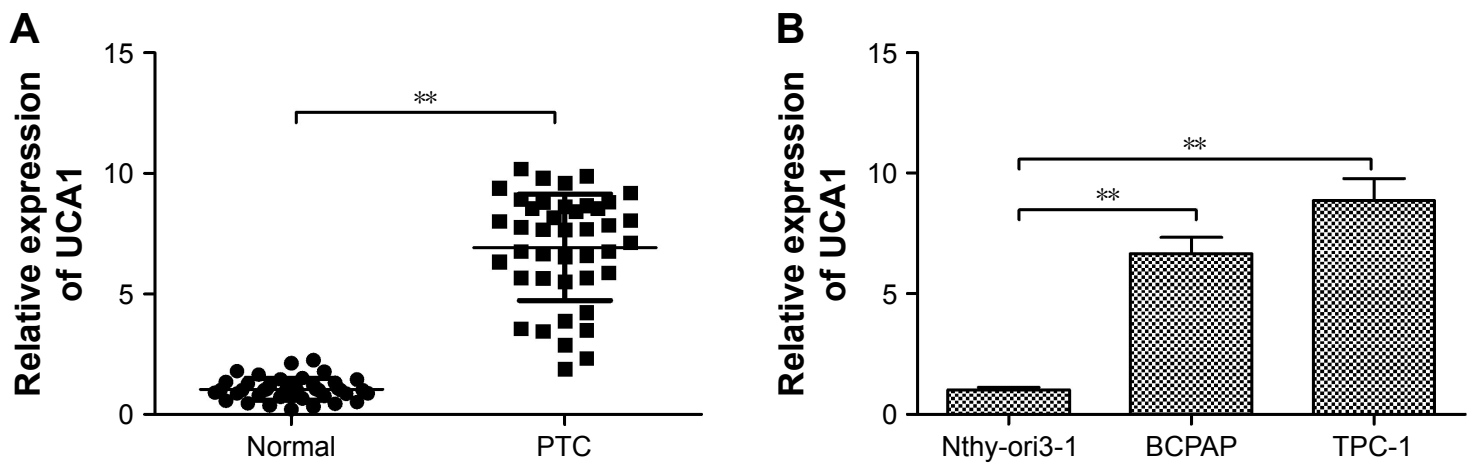

Figure I UCA I was upregulated in PTC tissues and cell lines.

Notes: (A) Relative UCAI expression in PTC tissues and adjacent normal tissues was determined by qRT-PCR ( $n=42)$. GAPDH was used as an internal control. (B) The expression levels of UCAI in two PTC cell lines (BCPAP and TPC-I) and normal thyroid cell line Nthy-ori3-I were examined by qRT-PCR. GAPDH was used as an internal control. All data are representative of three independent experiments and expressed as mean $\pm \mathrm{SD}$. **P $<0.0 \mathrm{I}$.

Abbreviations: PTC, papillary thyroid carcinoma; qRT-PCR, quantitative reverse transcription-PCR.

We found that the expression level of UCA1 was dramatically higher in PTC cell lines than that of Nthy-ori3-1 cells (Figure 1B).

\section{Knockdown of UCAI inhibits the progression of PTC}

To explore the effects of UCA1 expression on the progression of PTC, TPC-1 cells were transfected with UCA1 siRNAs (si-UCA1) and negative control (si-NC) for 48 hours using Lipofectamine 2000. qRT-PCR assay demonstrated that UCA1 was efficiently silenced in TPC-1 cells by si-UCA1 $(P<0.01$; Figure 2A). To investigate the effect of UCA1 on cell proliferation, CCK8 assay was performed. Our result demonstrated that knockdown of UCA1 inhibited the proliferation of TPC- 1 cells $(P<0.05$; Figure 2B). To further explore the effect of silencing UCA1 on the metastasis of cells, the migration and invasion were examined by wound-healing and transwell invasion assays, respectively. We found that knockdown of UCA1 significantly inhibited cell migration $(P<0.05$; Figure $2 \mathrm{C})$ and invasion $(P<0.05$; Figure 2D) in TPC-1 cells. These results suggested that knockdown of UCA1 suppresses PTC progression.

\section{UCAI serves as a competing endogenous RNA (ceRNA) and negatively regulates miR-204 expression in PTC}

It was well known that lncRNAs exerts it biological role in various types of cancer by acting as a ceRNA to sponge miRNAs. ${ }^{21}$ To explore the underlying mechanism of the growth inhibition by UCA1 knockdown in PTC, a bioinformatics analysis was adopted and revealed that UCA1 harbors a miR-204 recognition sequence (Figure 3A). Moreover, miR204 can decrease cell proliferation and invasion of PTC. ${ }^{22}$ Thus, we sought to confirm the potential association between UCA1 and miR-204 in PTC. A luciferase reporter assay was performed and revealed that overexpression of miR-204 suppressed the luciferase activity of the UCA1-Wt-3'-UTR, while no significant change was detected in luciferase activity of the UCA1-Mut-3'-UTR plasmid in TPC-1 cells (Figure 3B). Moreover, qRT-PCR was used to measure the expression level of miR-204 in TPC-1 cells transfected with si-UCA1 or si-NC. The results indicated that knockdown of UCA1 increased the expression level of miR-204 in TPC-1 cells $(P<0.05$; Figure 3C). Meanwhile, we found that upregulation of miR-204 significantly decreased UCA1 expression, whereas downregulation of miR-204 increased UCA1 expression in TPC-1 cells $(P<0.05$; Figure 3D).

The association between UCA1 and miR-204 was investigated to clarify the mechanism behind the regulatory role of UCA1 in PTC. We transfected si-NC, si-UCA1, and miR-204 inhibitor+si-UCA1 into TPC-1 cells; then, qRT-PCR was used to detect miR-204 expression. Specifically, miR-204 was upregulated in TPC-1 cells transfected with si-UCA1 compared to that of the si-NC group, and this upregulation was rescued by miR-204 inhibitor $(P<0.05$; Figure $3 \mathrm{E})$. In terms of function, we discovered that knockdown of UCA1 markedly inhibited the proliferation, migration, and invasion of TPC-1 cells $(P<0.05)$, which was rescued by transfection with the miR-204 inhibitor (Figure $3 \mathrm{~F}-\mathrm{H}$ ). These results suggested that the regulatory effect of UCA1 knockdown in PTC was counteract by miR-204 inhibitor. In other words, UCA1 promoted PTC progression through negative modulation of miR-204. 
A

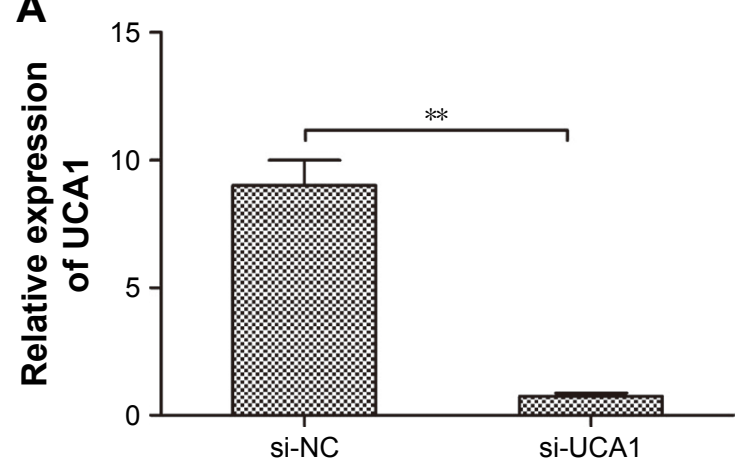

C
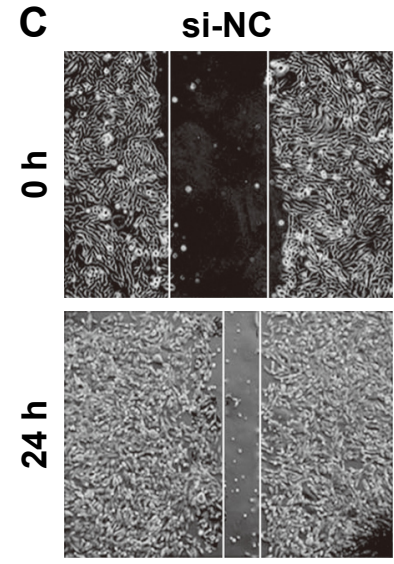

D
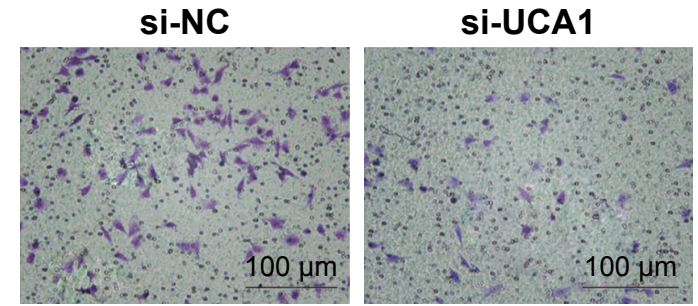

si-UCA1
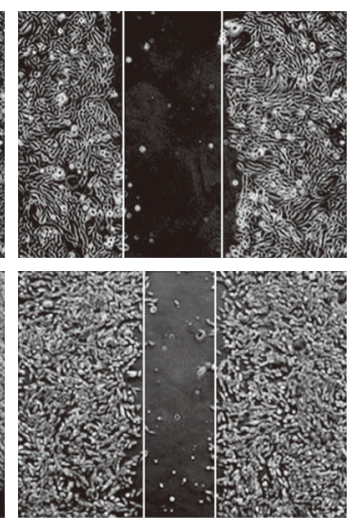

B
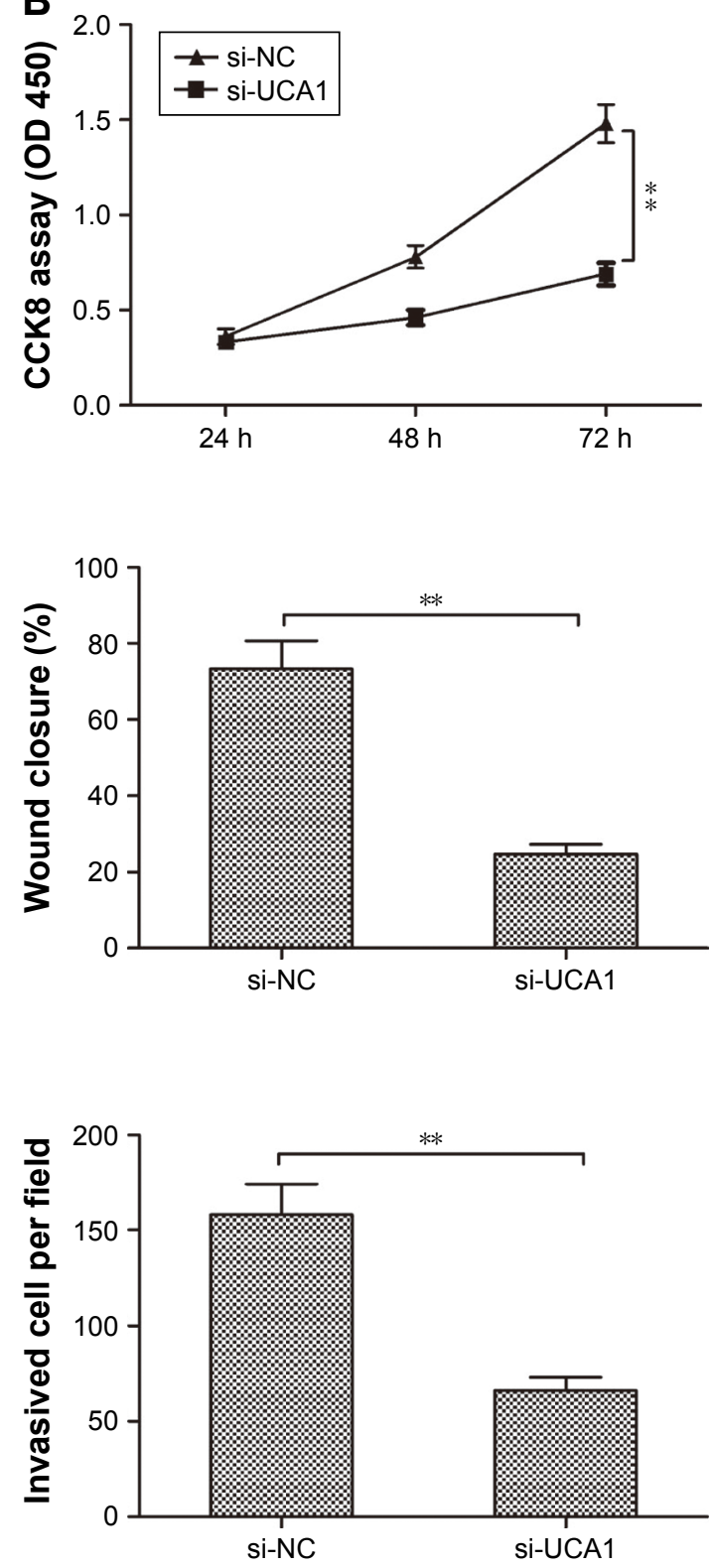

Figure 2 Knockdown of UCAI inhibited the PTC cell proliferation, migration, and invasion.

Notes: (A) Relative UCAI expression was detected in TPC-I cells transfected with si-UCAI or si-NC. GAPDH was used as an internal control. (B-D) Cell proliferation, migration, and invasion were determined in TPC-I cells transfected with si-UCAI or si-NC. All data are representative of three independent experiments and expressed as mean \pm SD. $* * P<0.01$.

Abbreviations: PTC, papillary thyroid carcinoma; NC, negative control.

\section{UCAI regulated IGFBP5 expression through miR-204}

Previously, a study demonstrated that miR-204-5p acts as a tumor suppressor in PTC by regulating IGFBP5. ${ }^{22}$ Thus, we wonder whether UCA1 could regulate IGFBP5 through miR-204, thus to act on PTC cell proliferation and invasion. We observed that knockdown of UCA1 could significantly inhibit IGFBP5 expression on mRNA and protein levels; the suppressive effect of UCA1 knockdown on IGFBP5 expression could be partially reversed by miR-204 inhibitor in TPC-1 cells (Figure 4A and B). Moreover, we also found that UCA1 expression was negatively correlated with miR-204 expression ( $r=-0.381, P=0.012$; Figure 4C), and positively correlated with IGFBP5 expression ( $r=0.547, P<0.001$; Figure 4D) in PTC tissues. These results showed that UCA1 regulated IGFBP5 expression by sponging to miR-204 expression in PTC cells.

\section{Discussion}

A number of lncRNAs have been shown to be involved in tumorigenesis and development of PTC and function 


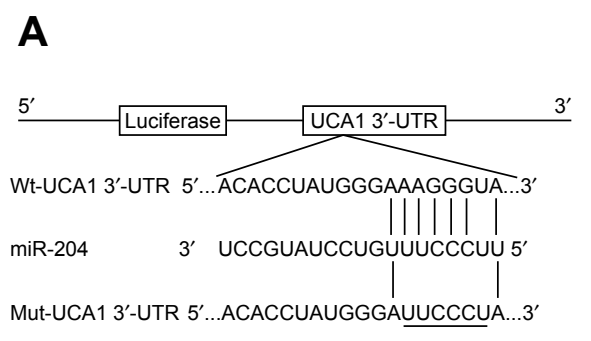

D

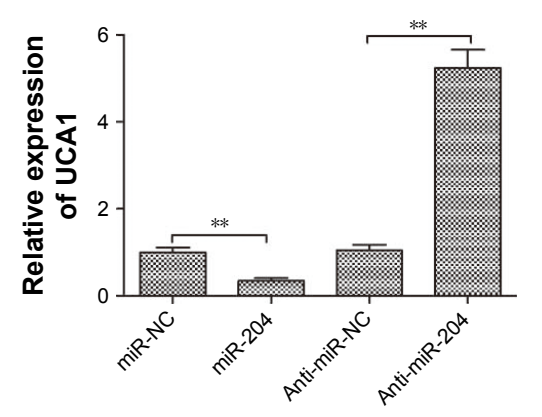

B

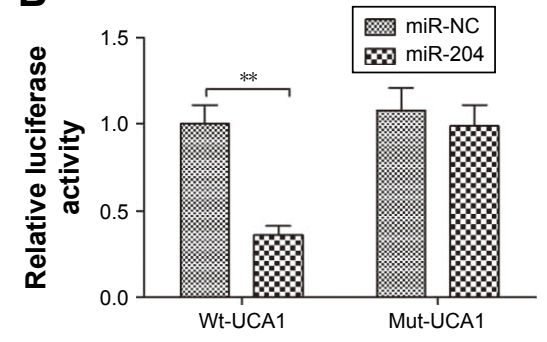

E

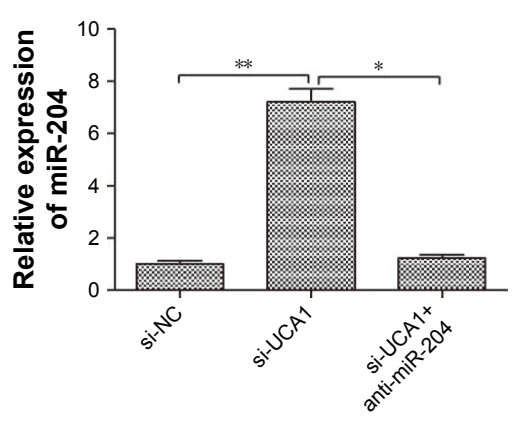

C

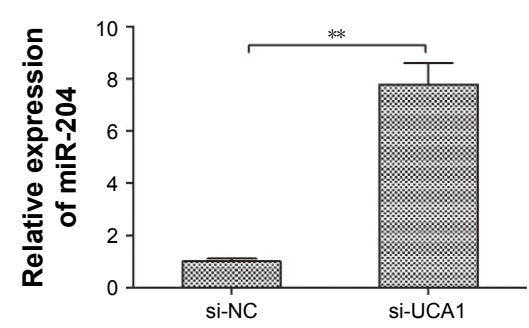

F

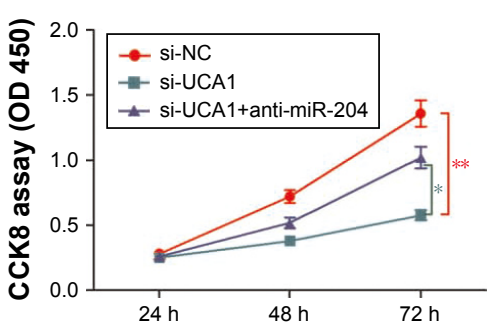

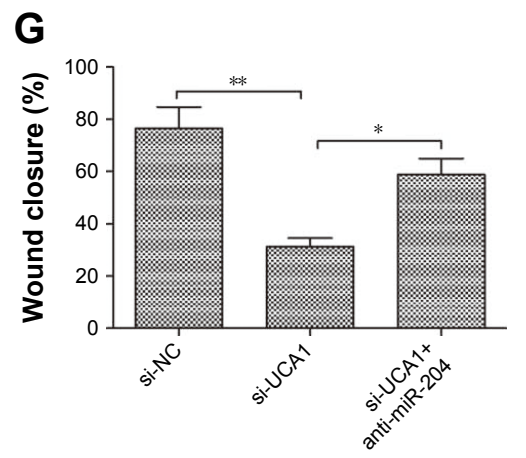

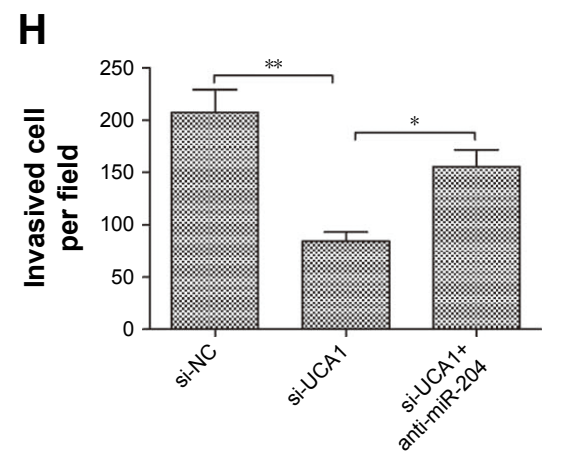

Figure 3 UCAI serves as a ceRNA and negatively regulates miR-204 expression in PTC.

Notes: (A) Schematic of the construction of Wt- or Mut-UCAI $3^{\prime}$-UTR vectors is indicated. (B) Relative luciferase activities were analyzed in TPC-I cells cotransfected with the Wt-UCAI or Mut-UCAI reporter plasmid and miR-204 mimic or miR-NC. (C) miR-204 expression level was determined in TPC-I cells transfected with si-UCAI or si-NC. U6 was used as an internal control. (D) UCAI expression level was determined in TPC-I cells transfected with miR-204 mimic, miR-NC, miR-204 inhibitor (antimiR-204), or anti-miR-NC. GAPDH was used as an internal control. (E) miR-204 expression level was determined in TPC-I cells transfected with si-NC or si-UCAI and with/without anti-miR-204. U6 was used as an internal control. (F-H) Cell proliferation, migration, and invasion were determined in TPC-I cells transfected with si-NC or si-UCAI and with/without anti-miR-204. All data are representative of three independent experiments and expressed as mean \pm SD. $* P<0.05$ and $* * P<0.01$.

Abbreviations: ceRNA, competing endogenous RNA; PTC, papillary thyroid carcinoma; Wt, wild type; Mut, mutant type; NC, negative control.

as oncogenes or tumor suppressor genes. ${ }^{10,11}$ Thus, more efforts should be made to deeply clarify the biological and molecular mechanisms of lncRNAs in PTC. In the present study, we found that UCA1 is upregulated in human PTC tissues and cell line (both $P<0.01$ ), and knockdown of UCA1 expression with si-UCA1 inhibits cell proliferation, migration, and invasion of TPC-1 cells. Therefore, we speculated that UCA1 might play a crucial role in the development and progression of PTC.

UCA1, located on chromosome 19p13.12, has been identified as an oncogene in multiple types of malignancies. ${ }^{12-20}$ For example, Xiao et $\mathrm{al}^{23}$ showed that UCA1 promoted hepatocellular carcinoma cells proliferation, invasion, and epithelial-mesenchymal transition (EMT) process in vitro and xenograft tumour growth in vivo by regulating miR-203/snail2 axis. Gu et al ${ }^{24}$ reported that UCA1 promoted cell proliferation, colony formation ability, and cell invasion in gastric cancer, as well as increased tumor growth in vivo by negatively regulating miR-590-3p. Zhang et a ${ }^{25}$ demonstrated that IncRNA UCA1 promoted migration and invasion in pancreatic cancer cells via the Hippo pathway. Consistent with these results, our data showed that UCA1 was significantly increased in PTC tissues and cell lines. Knockdown of UCA1 expression suppressed cell prolifera- 


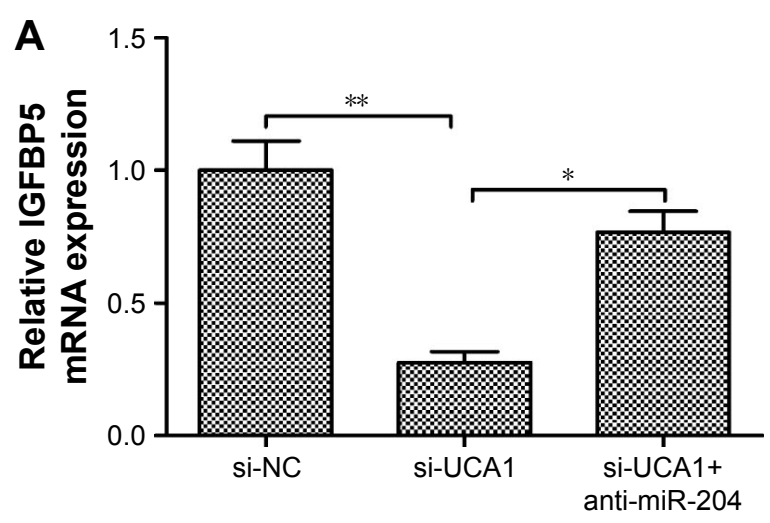

B
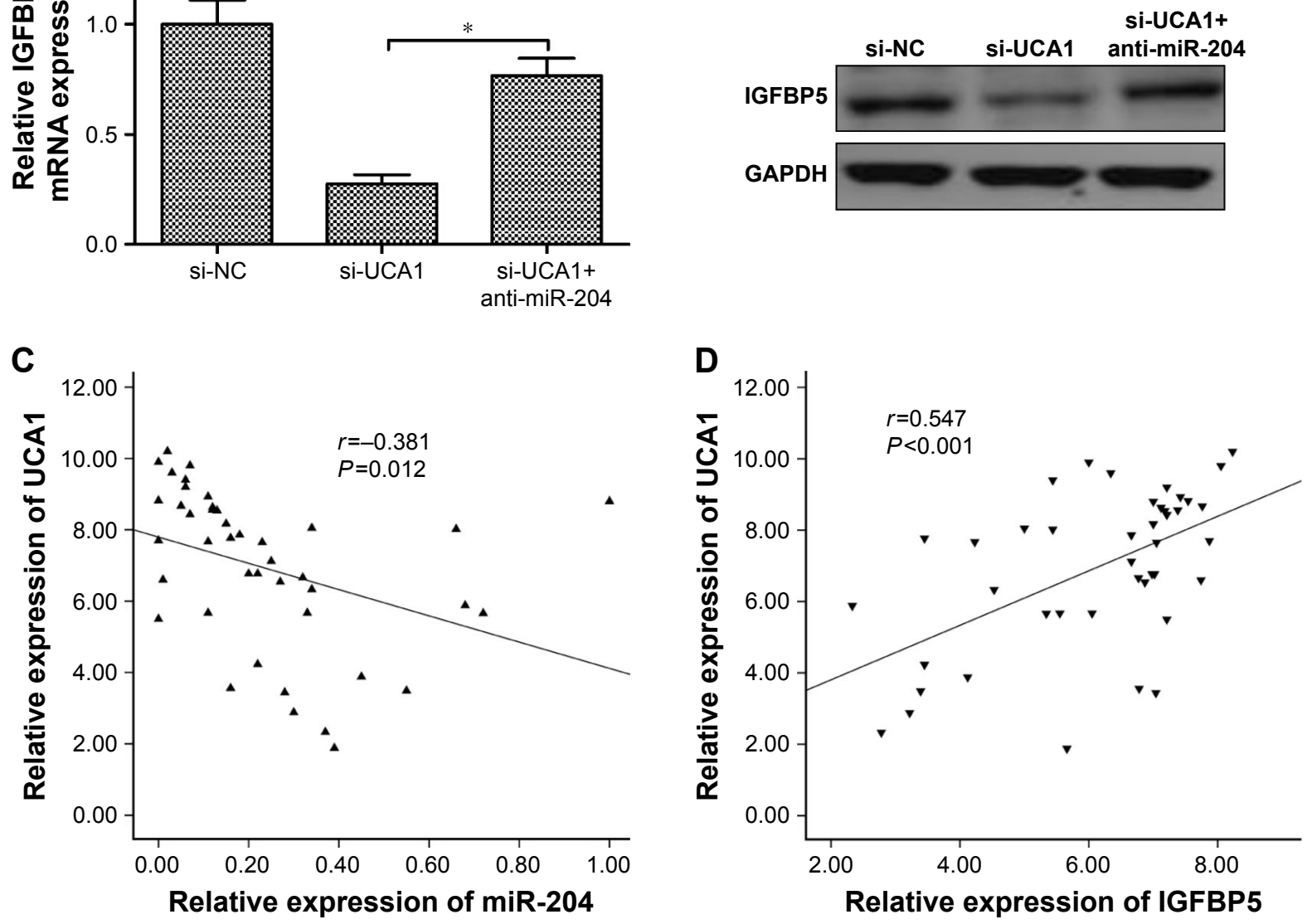

Figure 4 UCAI regulated IGFBP5 expression through miR-204.

Notes: (A) IGFBP5 mRNA expression was measured in TPC-I cells transfected with si-NC or si-UCAI and with/without anti-miR-204 by qRT-PCR. GAPDH was used as an internal control. (B) IGFBP5 protein expression was determined in TPC-I cells transfected with si-NC or si-UCAI and with/without anti-miR-204 by Western blot. GAPDH was used as an internal control. (C) UCAI expression levels were inversely correlated with the miR-204 level in 42 PTC samples using Pearson's correlation analysis. (D) UCAI expression levels were positively correlated with the IGFBP5 level in 42 PTC samples using Pearson's correlation analysis. All data are representative of three independent experiments and expressed as mean $\pm S D$. $* P<0.05$ and $* * P<0.01$.

Abbreviations: NC, negative control; qRT-PCR, quantitative reverse transcription-PCR; PTC, papillary thyroid carcinoma.

tion, migration, and invasion. Therefore, our findings suggested that UCA1 could act as an oncogene and a potential therapeutic target for PTC.

Accumulating evidence has been suggested that lncRNA can serve as ceRNA abrogating the endogenous suppressive effect of these miRNAs on their targeted transcripts. ${ }^{21}$ UCA1 has been shown to harbor the recognition sequence of many miRNAs, such as miR-27b, ${ }^{17} \mathrm{miR}-204-5 \mathrm{p},{ }^{18} \mathrm{miR}-$ 193-3 p, ${ }^{20} \mathrm{miR}-203,{ }^{23} \mathrm{miR}-590-3 \mathrm{p},{ }^{24}$ and miR-96. ${ }^{26}$ Thus, for further investigation on the specific regulatory mechanism of UCA1 in PTC, we identified its downstream target miR204, which has generally been reported as a tumor suppressor in PTC. ${ }^{22}$ In addition, miR-204 has been confirmed to be a downstream target of UCA1 in colorectal cancer cells. ${ }^{18}$ Consistent with previous result, we further identified miR-204 as a direct target of UCA1 in PTC by luciferase activity assay. Furthermore, we observed that knockdown of UCA1 can increase miR-204 expression, whereas downregulation of miR-204 increased UCA1 expression and upregulation of miR-204 decreased UCA1 expression. Importantly, we revealed that the inhibition effect of UCA1 in PTC was counteracted by miR-204 inhibitor. These results suggested that UCA1 promoted PTC cell proliferation, migration, and invasion by negatively regulating miR-204.

A previous report has indicated that miRNA-204 inhibits proliferation and invasion in PTC by targeting IGFBP5. ${ }^{22}$ IGFBP5 has been reported to be involved in PTC progression and function as an oncogene. ${ }^{22,27}$ Thus, we wonder whether UCA1 could regulate IGFBP5 through miR-204, thus to act on PTC cell proliferation and invasion. In the present study, we found that knockdown of UCA1 inhibits IGFBP5 expression, while co-transfection of miR-204 inhibitor and 
si-UCA1 reversed the decreased IGFBP5 expression induced by si-UCA1. Moreover, we also found that UCA1 expression was positively correlated with IGFBP5 expression in PTC. These results indicated that UCA1/miR-204/IGFBP5 was responsible for the progression of PTC cells.

\section{Conclusion}

Our studies indicated that UCA1 could promote PTC progression. Mechanic analysis revealed the involvement of UCA1 in promoting PTC progression by functioning as miR-204 sponge and suggested a novel UCA1/miR-204/ IGFBP5 signaling pathway regulatory network in PTC. These results suggested that UCA1 may serve as a potential target for PTC treatment.

\section{Disclosure}

The authors report no conflicts of interest in this work.

\section{References}

1. Chen W, Zheng R, Zeng H, Zhang S, He J. Annual report on status of cancer in China, 2011. Chin J Cancer Res. 2015;27(1):2-12.

2. Guilmette J, Nosé V. Hereditary and familial thyroid tumours. Histopathology. 2018;72(1):70-81.

3. Lombardi D, Accorona R, Paderno A, Cappelli C, Nicolai P. Morbidity of central neck dissection for papillary thyroid cancer. Gland Surg. 2017; 6(5):492-500.

4. Morlando M, Fatica A. Alteration of Epigenetic Regulation by Long Noncoding RNAs in Cancer. Int J Mol Sci. 2018;19(2):570.

5. Mercer TR, Dinger ME, Mattick JS. Long non-coding RNAs: insights into functions. Nat Rev Genet. 2009;10(3):155-159.

6. Schaukowitch K, Kim TK. Emerging epigenetic mechanisms of long non-coding RNAs. Neuroscience. 2014;264:25-38.

7. Sun M, Kraus WL. From discovery to function: the expanding roles of long noncoding RNAs in physiology and disease. Endocr Rev. 2015; 36(1):25-64.

8. Chen L, Dzakah EE, Shan G. Targetable long non-coding RNAs in cancer treatments. Cancer Lett. 2018;418:119-124.

9. Zhang Y, Tang L. The Application of IncRNAs in Cancer Treatment and Diagnosis. Recent Pat Anticancer Drug Discov. 2018;13(3):292-301.

10. Sui F, Ji M, Hou P. Long non-coding RNAs in thyroid cancer: Biological functions and clinical significance. Mol Cell Endocrinol. 2018;469:11-22.

11. Murugan AK, Munirajan AK, Alzahrani AS. Long noncoding RNAs: emerging players in thyroid cancer pathogenesis. Endocr Relat Cancer. 2018;25(2):R59-R82.
12. Wang XS, Zhang Z, Wang HC, et al. Rapid identification of UCA1 as a very sensitive and specific unique marker for human bladder carcinoma. Clin Cancer Res. 2006;12(16):4851-4858.

13. Liu H, Wang G, Yang L, Qu J, Yang Z, Zhou X. Knockdown of Long Non-Coding RNA UCA1 Increases the Tamoxifen Sensitivity of Breast Cancer Cells through Inhibition of Wnt/B-Catenin Pathway. PLoS One. 2016;11(12):e0168406.

14. Fotouhi Ghiam A, Taeb S, Huang X, et al. Long non-coding RNA urothelial carcinoma associated 1 (UCA1) mediates radiation response in prostate cancer. Oncotarget. 2017;8(3):4668-4689.

15. Jiao C, Song Z, Chen J, et al. IncRNA-UCA1 enhances cell proliferation through functioning as a ceRNA of Sox 4 in esophageal cancer. Oncol Rep. 2016;36(5):2960-2966.

16. Chen P, Wan D, Zheng D, Zheng Q, Wu F, Zhi Q. Long non-coding RNA UCA1 promotes the tumorigenesis in pancreatic cancer. Biomed Pharmacother. 2016;83:1220-1226.

17. Fang Q, Chen X, Zhi X. Long Non-Coding RNA (LncRNA) Urothelial Carcinoma Associated 1 (UCA1) Increases Multi-Drug Resistance of Gastric Cancer via Downregulating miR-27b. Med Sci Monit. 2016; 22:3506-3513.

18. Bian Z, Jin L, Zhang J, et al. LncRNA-UCA1 enhances cell proliferation and 5 -fluorouracil resistance in colorectal cancer by inhibiting miR-204-5p. Sci Rep. 2016;6:23892.

19. Yang Y, Jiang Y, Wan Y, et al. UCA1 functions as a competing endogenous RNA to suppress epithelial ovarian cancer metastasis. Tumour Biol. 2016;37(8):10633-10641.

20. Nie W, Ge HJ, Yang XQ, et al. LncRNA-UCA1 exerts oncogenic functions in non-small cell lung cancer by targeting miR-193a-3p. Cancer Lett. 2016;371(1):99-106.

21. Chan J, Tay Y. Noncoding RNA:RNA Regulatory Networks in Cancer. Int J Mol Sci. 2018;19(5):E1310.

22. Liu L, Wang J, Li X, et al. MiR-204-5p suppresses cell proliferation by inhibiting IGFBP5 in papillary thyroid carcinoma. Biochem Biophys Res Commun. 2015;457(4):621-626.

23. Xiao JN, Yan TH, Yu RM, et al. Long non-coding RNA UCA1 regulates the expression of Snail2 by miR-203 to promote hepatocellular carcinoma progression. J Cancer Res Clin Oncol. 2017;143(6): 981-990.

24. Gu L, Lu LS, Zhou DL, Liu ZC. UCA1 promotes cell proliferation and invasion of gastric cancer by targeting CREB1 sponging to miR-590-3p. Cancer Med. 2018;7(4):1253-1263.

25. Zhang M, Zhao Y, Zhang Y, et al. LncRNA UCA1 promotes migration and invasion in pancreatic cancer cells via the Hippo pathway. Biochim Biophys Acta. 2018;1864(5 Pt A):1770-1782.

26. Zhou Y, Chen Y, Ding W, et al. LncRNA UCA1 impacts cell proliferation, invasion, and migration of pancreatic cancer through regulating miR-96/FOXO3. IUBMB Life. 2018;70(4):276-290.

27. Stolf BS, Carvalho AF, Martins WK, et al. Differential expression of IGFBP-5 and two human ESTs in thyroid glands with goiter, adenoma and papillary or follicular carcinomas. Cancer Lett. 2003;191(2): 193-202.
OncoTargets and Therapy

\section{Publish your work in this journal}

OncoTargets and Therapy is an international, peer-reviewed, open access journal focusing on the pathological basis of all cancers, potential targets for therapy and treatment protocols employed to improve the management of cancer patients. The journal also focuses on the impact of management programs and new therapeutic agents and protocols on
Dovepress

patient perspectives such as quality of life, adherence and satisfaction. The manuscript management system is completely online and includes a very quick and fair peer-review system, which is all easy to use. Visit http://www.dovepress.com/testimonials.php to read real quotes from published authors. 